\title{
Stage R
}

National Cancer Institute

\section{Source}

National Cancer Institute. Stage R. NCI Thesaurus. Code C139577.

A general term that refers to the histopathological staging of residual tumors after treatment. 